\title{
Evaluation of Smart Antenna Algorithms for cdma2000 Reverse Link
}

\author{
Mustafa Karakoc ${ }^{1}$ and Adnan Kavak ${ }^{2}$ \\ ${ }^{1}$ Kocaeli University, Dept. of Electronics and Computer Ed., 41100, Kocaeli, Turkey \\ ${ }^{2}$ Kocaeli University, Dept. of Computer Engineering, 41040, Kocaeli, Turkey \\ \{mkarakoc, akavak\} @kou.edu.tr
}

\begin{abstract}
Adaptive algorithms which provide desired performance and have small computational load are key factors that enable the integration of smart antennas into $3 \mathrm{G}$ wireless communication systems. We evaluate non-blind (LMS, RLS), blind (CM), and semi-blind (CFA) type receive beamforming algorithms in terms of their performance, complexity, and convergence rate for cdma2000 systems. Simulations are performed for a 5-element uniform linear array operating in a wireless vector channel that consists of multipaths and multiple access interference. Results show that CFA has the highest SINR with minimum deviation in varying fading conditions. CM has the smallest SINR performance possibly due to not involving pilot information, but it has the computation load as low as LMS does, i.e. $\mathrm{O}(\mathrm{M})$ where $\mathrm{M}$ is the antenna number. RLS achieves SINR comparable to CFA with the fastest convergence rate.
\end{abstract}

\section{Introduction}

With the emergence of the third generation $(3 \mathrm{G})$ wireless systems, smart antenna systems (SAS) which are proven to provide capacity increase and coverage expansion becomes an attractive technology. The heart of the SAS is their advanced signal processing capability at the baseband, which enables them to adaptively adjust their beam pattern. In order to deploy SAS in $3 G$ systems, it is, however, essential to employ beamforming algorithms which provide desired performance and have small computational complexity to be implemented in real time.

In this paper, we evaluate blind type Constant Modulus (CM) [1], semi-blind type Code Filtering Approach (CFA) [2], and non-blind type Least Mean Square (LMS) and Recursive Least Squares (RLS) [3,4] adaptive beamforming algorithms from various aspects for cdma2000 system in changing channel propagation conditions. The objective function common to these algorithms is that they try to maximize signal-to-interference plus noise ratio (SINR) at the output of the beamformer. However, they differ in the computation of beamforming weight vector that approaches Wiener solution. In obtaining optimum weight vector, while LMS, RLS, and CM algorithms use iterative approaches, CFA solves generalized eigenvalue problem using the estimated covariance matrices of the array output and postcorrelation signal vector. In the non-blind type adaptive algorithms, a training signal is known to both the transmitter and receiver during the training period issued. On the 
other hand, the blind type adaptive algorithm does not require any training sequence and exploit some known properties of the desired signal, such as discrete-alphabet structure, constant-modulus property or direction-of-arrivals (DOAs) information. Semi-blind type adaptive algorithm overcomes the problem of insufficient training symbols of non-blind methods and the requirement of large amount of data as in blind methods.

Simulations are performed for the reverse link of cdma2000 under varying multipath and multiple access interference conditions. The results to be presented include the comparison of SINR variation, computation complexity, DOA estimation error, and convergence time for the above algorithms.

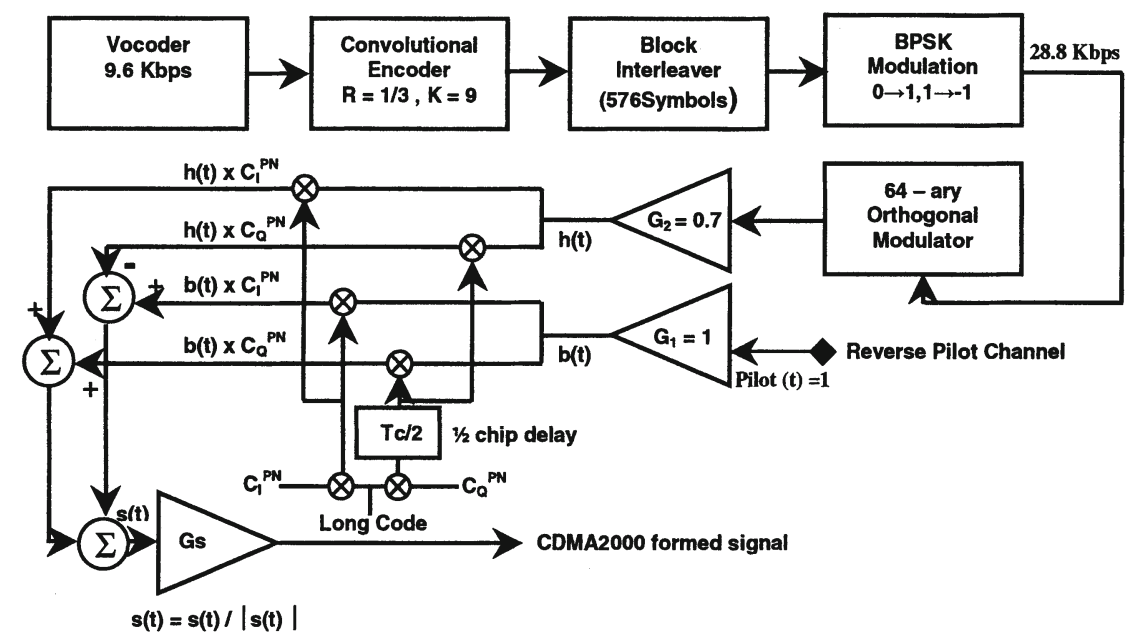

Fig. 1. Reverse link of the cdma2000 system for RC1

\section{Uplink Signal Model}

Cdma2000 is an approved 3G standard by ITU, which is based on the existing IS-95 CDMA system. [5, 6]. The cdma2000 reverse link baseband signal model in radio configuration 1 (RC1) is used in our simulations as shown in Figure 1. The transmitted baseband signal $\mathrm{s}(\mathrm{t})$ from a mobile unit can be expressed as,

$$
\mathrm{s}(\mathrm{t})=\sum_{k=1}^{\infty} \mathrm{d}(\mathrm{k}, \mathrm{t}) \otimes \mathrm{c}(\mathrm{k}, \mathrm{t})
$$

where $\mathrm{k}$ is the slot index, $\mathrm{d}(\mathrm{k}, \mathrm{t})$ is the waveform before complex spreading, $\mathrm{c}(\mathrm{k}, \mathrm{t})$ is the complex PN spreading sequence, $\otimes$ denotes the complex spread function. The waveform before complex spreading $\mathrm{d}(\mathrm{k}, \mathrm{t})$ is composed of the traffic channel and pilot channel information symbols, which are given by

$$
d(k, t)=d_{I}(k, t)+j d_{Q}(k, t)
$$

where $\mathrm{j}$ represents the imaginary part of Eq. 2, and 


$$
\begin{aligned}
& \mathrm{d}_{\mathrm{I}}(\mathrm{k}, \mathrm{t})=\sum_{\mathrm{i}=1}^{\mathrm{N}_{\mathrm{C}}} \mathrm{G}_{1} \mathrm{~b}\left((\mathrm{k}-1) \mathrm{N}_{\mathrm{C}}+\mathrm{i}\right), \\
& \mathrm{d}_{\mathrm{Q}}(\mathrm{k}, \mathrm{t})=\sum_{\mathrm{i}=1}^{\mathrm{N}_{\mathrm{C}}} \mathrm{G}_{2} \mathrm{~h}\left((\mathrm{k}-1) \mathrm{N}_{\mathrm{C}}+\mathrm{i}\right),
\end{aligned}
$$

where $b(\cdot)$ is the bit stream of pilot channel which are all " 1 "s, $d(\cdot)$ is the bit stream of traffic channel and $\mathrm{N}_{\mathrm{c}}$ is the length of spreading code per slot. The complex PN spreading sequence waveform is

$$
\mathrm{c}(\mathrm{k}, \mathrm{t})=\sum_{\mathrm{j}=0}^{\mathrm{N}_{\mathrm{c}}-1} \mathrm{C}^{\mathrm{PN}} \mathrm{p}\left(\mathrm{t}-\left[(\mathrm{k}-1) \mathrm{N}_{\mathrm{c}}+\mathrm{j}\right] \mathrm{T}_{\mathrm{c}}\right),
$$

where $\mathrm{T}_{\mathrm{c}}$ is the chip period and $C^{P N}$ is the complex spreading code whose real and imaginary parts are identically distributed, random binary numbers taken from the set $\{+1,-1\}$ with equal probability. The transmitted signal $s(t)$ is exposed to multipath propagation environment, which induces complex path attenuation, $\alpha_{l}=\beta_{l} e^{j \phi_{l}}$ and time delay $\tau_{\ell}$ to each multipath signal. The signal received by $\mathrm{M}$ element antenna array at the base station can be written as,

$$
\mathbf{X}(\mathrm{t})=\sum_{\ell=1}^{L} \alpha_{\ell} \mathrm{s}\left(\mathrm{t}-\tau_{\ell}\right) \mathbf{a}\left(\theta_{\ell}\right)+\mathbf{I}(\mathrm{t})+\mathbf{N}(\mathrm{t}),
$$

where $\mathbf{I}(t)$ is the multiple access interference (MAI) which is given by

$$
\mathbf{I}(\mathrm{t})=\sum_{q=1}^{N-1} \sum_{\ell=1}^{L_{q}} \alpha_{q, \ell} \mathrm{s}\left(\mathrm{t}-\tau_{\mathrm{q}, \ell}\right) \mathbf{a}\left(\theta_{\mathrm{q}, \ell}\right)
$$

and $\mathbf{N}(\mathrm{t})$ is the Mx1 complex-valued additive spatially white Gaussian noise vector, $\mathbf{a}\left(\theta_{\ell}\right)$ is the Mx1 array response vector of the multipath arriving at DOA $\theta_{\ell}$. Finally, the array output is multiplied by a complex weight vector $\mathrm{W}$ which is determined according to aforementioned beamforming algorithms to result in

$$
\mathbf{z}(\mathrm{t})=\mathbf{w}^{H}(\mathrm{t}) \mathbf{X}(\mathrm{t}),
$$

where $H$ denotes complex conjugation and transpose (Hermitian) operation.

\section{SINR Calculation}

SINR of the received signal at the beamformer output is calculated using the similar approach described in [2]. The post-correlation signal vector for the multipath signal of the desired user can be written as 


$$
\mathbf{y}_{\mathbf{1}}(t)=\frac{1}{\sqrt{T_{b}}} \int_{(t-1) T_{c}}^{t T_{c}} \mathbf{X}(t) \mathrm{c}_{1}^{*}\left(t-\tau_{1}\right) d t
$$

where, $T_{b}$ is the symbol duration, $c_{1}$ is the code of desired user, $\tau_{1}$ is the time delay for the desired multipath signal. In order to find the SINR, it is necessary to first estimate the pre-correlation $\mathbf{R}_{\mathrm{xx}}$ and post-correlation $\mathbf{R}_{\mathrm{yy}, 1}$ covariance matrices of the signal vectors $\mathbf{X}(\mathrm{t})$ and $\mathbf{y}_{1}(\mathrm{t})$ in (7) and in (9), respectively. The interference-plus-noise covariance matrix $\mathbf{R}_{\mathrm{uu}, \mathrm{l}}$ can be estimated utilizing these matrices as given by

$$
\mathbf{R}_{\mathrm{uu}, \mathrm{l}}=\frac{\mathrm{G}}{\mathrm{G}-1}\left(\mathbf{R}_{\mathrm{xx}}-\frac{1}{\mathrm{G}} \mathbf{R}_{\mathrm{yy}, \mathrm{l}}\right),
$$

where, $G$ is the processing gain defined as $G=T_{b} / T_{c}$. Then, the covariance matrix for the desired signal component $s_{1}(t)$ is estimated from

$$
\mathbf{R}_{\mathrm{ss}}=\mathbf{R}_{\mathrm{yy}, 1}-\mathbf{R}_{\mathrm{uu}, 1} \text {. }
$$

Finally, using the optimum weight vector generated at the output of each beamforming algorithm, we can calculate the received SINR

$$
\mathrm{SINR}=\frac{\mathbf{w}_{\mathrm{opt}}^{*} \mathbf{R}_{\mathrm{ss}, 1} \mathbf{w}_{\mathrm{opt}}}{\mathbf{w}_{\mathrm{opt}}^{*} \mathbf{R}_{\mathrm{uu}, 1} \mathbf{w}_{\mathrm{opt}}}
$$

\section{Simulations and Results}

We consider a multipath propagation scenario that has a direct path signal at $32^{\circ}$ and two multipath signals at $44^{\circ}$ and $85^{\circ}$ for the desired user and an interference signal at $61^{\circ}$. The base station antenna configuration is 5-element uniform linear array (ULA). We perform repeated simulations (100 times) in order to compute the average SINR for each algorithm under varying channel fading conditions. These 100 runs represent that we take 100 spatial points as the mobile moves a small distance along a given direction. Therefore, as demonstrated in [7], DOAs $\left(\theta_{l}\right)$ are assumed to remain unchanged during this small movement. For each simulation, the antenna array receives 1000 signal samples and executes adaptive algorithms. We assume that Rayleigh random variable represents the corresponding amplitude fade $\left(\beta_{l}\right)$ along the multipath. For the phase component $\left(\phi_{l}\right)$ of complex path attenuation at each multipath, uniformly distributed random variable is assumed, which represents path length difference and Doppler shift in the propagation medium. Fading parameters in the channel are adjusted such that direct path signal is minimum $3 \mathrm{~dB}$ above the multipaths and $5 \mathrm{~dB}$ above the interference. Multipath time delays are set as one chip period $\left(T_{c}\right)$ for $85^{\circ}$ and half chip period $\left(T_{c} / 2\right)$ for $44^{\circ}$. Convergence criteria for the LMS, RLS, and CM algorithms are determined from the norm of weight error vector given by

$$
\|\Delta \mathbf{w}\|=\|\mathbf{w}(t+1)-\mathbf{w}(t)\|,
$$


which is 0,0001 . Other relevant parameters for the simulations are chosen as following; step size parameter $\mu=0.01$ for LMS and CM; weight factor $\psi=0.99$ for $\mathrm{CM}$; forgetting factor $\delta_{\mathrm{o}}=0.99$ for RLS; code parameter $\eta=1.5$ and spreading gain $\mathrm{G}=4$ for CFA.

During simulations, CFA, CM, RLS, and LMS algorithms are assumed to run parallel on the received signal vector $\mathbf{X}(\mathrm{t})$ and each generates a weight vector based on the calculations given in $[1,2,3,4]$. Spatial spectrums formed with the weight vector resulting from these algorithms are plotted in Figures 2, 3, 4, and 5 for LMS, RLS, $\mathrm{CM}$, and CFA, respectively. Note that these figures are the results of a single simulation run, which are representative of 100 simulation runs. In each figure, we see that main beam direction is steered towards to DOA of desired signal's direct path. This is because the direct path signal was the strongest and the algorithms lock on the signal which has the largest power,

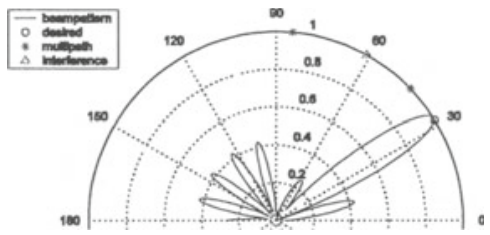

Fig. 2. Spatial spectrum via LMS alg.

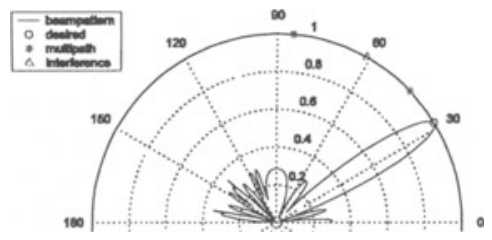

Fig. 4. Spatial spectrum via $\mathrm{CM}$ alg.

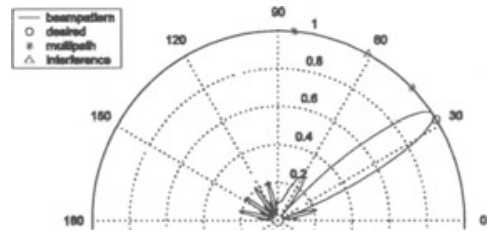

Fig. 3. Spatial spectrum via RLS alg.

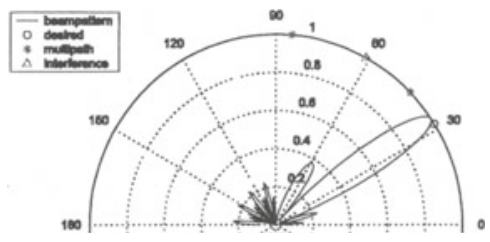

Fig. 5. Spatial spectrum via CFA alg.

Other results of the simulations are summarized in Table 1 . Note that the mean $(50 \%$ values) and standard deviation values of SINR are compiled from its cumulative distribution. CFA has the largest SINR among the four algorithms. It also has the smallest SINR deviation, implying the stability of this algorithm in changing channel conditions. RLS provides SINR value comparable to CFA, but it has the advantage of minimum convergence time. CFA is the most computationally complex algorithm with the order of $\mathrm{O}\left(\mathrm{M}^{3}\right)$ due to matrix inversion operation. The SINR performance of the $\mathrm{CM}$ is very low $(5 \mathrm{~dB})$ as compared to other three algorithms. This may be due to not utilizing additional pilot information provided by cdma2000 system as a training signal in $\mathrm{CM}$ algorithm. The only advantage of $\mathrm{CM}$ is its simplicity in the computation load in the order of $\mathrm{O}(\mathrm{M})$, which is as small as LMS algorithm. 
Table 1. Simulation results.

\begin{tabular}{|c|c|c|c|c|c|}
\hline \multicolumn{2}{|c|}{ ADAPTIVE ALGORITHMS } & LMS & RLS & $\mathbf{C M}$ & CFA \\
\hline \multirow{2}{*}{$\begin{array}{c}\text { Computational } \\
\text { complexity }\end{array}$} & add & $2 \mathrm{M}+1$ & $2 \mathrm{M}^{2}+\mathrm{M}$ & $2 \mathrm{M}+4$ & $\mathrm{M}^{3}+2 \mathrm{M}^{2}-1$ \\
\hline & mply & $2 \mathrm{M}$ & $5 \mathrm{M}^{2}+3 \mathrm{M}+1$ & $2 \mathrm{M}$ & $\mathrm{M}^{3}+3 \mathrm{M}^{2}+2 \mathrm{M}+3$ \\
\hline \multicolumn{2}{|c|}{ DOA estimation error (\%) } & 1,5625 & 6,250 & 0,6250 & 3,125 \\
\hline \multicolumn{2}{|c|}{ Convergence time } & 283 & 24 & 546 & (1) \\
\hline \multirow{2}{*}{$\begin{array}{c}\text { SINR } \\
(d B) \\
\end{array}$} & mean & 7,4565 & 11,8799 & 4,6018 & 12,056 \\
\hline & std. dev. & 0,57 & 0,65 & 3,08 & 0,05 \\
\hline
\end{tabular}

(1) Algorithm does not require iterative computation

\section{Conclusion}

We have found the following as a result of evaluating various receive beamforming algorithms for cdma2000 under changing channel conditions. We find that CFA is the robust algorithm providing the highest SINR $(\sim 12.1 \mathrm{~dB})$ among the four algorithms compared. The SINR performance of CFA is stable with minimum standard deviation. In terms of convergence time, RLS is the fastest algorithm. It also has the SINR performance $(\sim 11.9 \mathrm{~dB})$ as high as CFA does. The SINR performance of CM is the worst $(\sim 4.6 \mathrm{~dB})$ due to its blind type adaptation that possibly can not follow varying channel conditions. However, the results obtained herein need further verification for vehicular propagation conditions including the other beamforming methods such as DOA based or covariance matrix based beamforming.

Acknowledgement. This research was supported by TUBITAK (Scientific and Technical Research Council of Turkey) under contract EEEAG/102E015.

\section{References}

1. Veen, A. J., Paulraj, A.: An analytical constant modulus algorithm. IEEE Trans. of Signal Proc., 44(5) (1996) 1-19.

2. Naguib, A. F.: Adaptive antennas for CDMA wireless networks. Ph.D dissertation, Stanford Univ., (1996).

3. Rong, Z.: Simulation of adaptive array algorithms for CDMA systems. MS. Thesis, Virginia PI \& S Univ., (1996).

4. Godara, L.: Application of antenna arrays to mobile communications, Part II: beamforming and direction-of-arrival considerations. Proc. IEEE, Vol. 85. (1997) 11951245.

5. TIA/EIA Interim Standard, Physical Layer Standard for cdma2000 spread spectrum systems, TIA/EIA/S-2000-2.

6. Wideband cdmaOne (TIA cdma2000) Radio Transmission Technology Proposal. International Telecommunication Union, Radio communication Study Groups (1998) http://www.cdg.org/frame_3giis.html.

7. Kavak, A., Yang, W., Xu, G., Vogel, W. J.: Characteristics of vector propagation channels in dynamic mobile scenarios. IEEE Trans. on Antennas and Prop., 49(12) (2001) 16951703. 